\title{
Optical Amplification and Photosensitivity in Sol-Gel Based Waveguides
}

\author{
A. Selvarajan, Member, IEEE, and T. Srinivas, Member, IEEE
}

Invited Paper

\begin{abstract}
The sol-gel process has emerged as an effective route for the fabrication of optical waveguides and guided wave devices and circuits. In particular, it is possible to incorporate active dopants like neodymium, erbium, and cesium for integrated optical active devices and circuits. In this paper, a review of recent research on active devices and circuits based on sol-gel process is made. Specific studies undertaken in our laboratory on optical amplification and photosensitivity characteristics of sol-gel optical films are presented. In the case of modeling and analysis of active sol-gel films for the study of optical amplifiers, we present the Atomic Susceptibility Theory of lasers and also the method of rate equations, duly taking into account the waveguide parameters. The Beam Propagation Method is applied to study the propagation and gain characteristics of actively doped sol-gel film devices such as straight waveguide, $Y$-branch, and directional coupler. Formation of Bragg gratings in cerium-doped films are investigated. Also, futuristic applications in MEMS where the sol-gel process will be effectively used to form optical layers that can be controlled by mechanical effects are pointed out.
\end{abstract}

Index Terms-Atomic susceptibility theory, integrated optics, optical amplification, sol-gel waveguides, waveguide Bragg grating.

\section{INTRODUCTION}

$\mathbf{T}$ HE SOL-GEL process is a very simple and effective method for fabricating integrated optical devices [1] where films with varying composition can be easily prepared. Combined with lithographic process, this process can be used to realize several configurations of integrated optical devices can be realized. One of the advantages of the sol-gel process that can be effectively exploited is the ease with which dopants can be introduced. In particular, this can be employed to dope active materials such as neodymium, erbium, and cerium to fabricate integrated optical (IO) devices with amplifying and photosensitive properties. The incorporation of active materials, such as neodymium $\left(\mathrm{Nd}^{3+}\right)$ and erbium $\left(\mathrm{Er}^{3+}\right)$ in host media has potential applications in lasers and amplifiers, while cerium $\left(\mathrm{Ce}^{3+}\right)$ doping results in better photosensitivity.

This paper presents a review of the sol-gel process with a view to exploit it for fabricating optical amplifiers and other

Manuscript received December 4, 2000; revised May 22, 2001. This work was supported by the University Grants Commission (UGC), the Space Technology Cell (STC), and the Aeronautical Research and Development Board (ARDB).

The authors are with the Department of Electrical Communication Engineering, Indian Institute of Science, Bangalore 560012, India (e-mail rajan@ece.iisc.ernet.in).

Publisher Item Identifier S 0018-9197(01)07359-6. waveguide devices. It also presents some of our own analytical and experimental work [2]. A review of the recent literature pertaining to fiber amplifiers, waveguide amplifiers by non-sol-gel route, and waveguide amplifiers by the sol-gel process, is given in Section II. This section also includes a review on photosensitivity in fibers and planar waveguides. The next section deals with the analysis of waveguide amplifiers and devices and gives numerical simulations using parameters corresponding to sol-gel processed waveguides. Experimental work done in our laboratory in forming sol-gel films with amplifying dopants and photo-sensitive dopants are presented in Section IV. The concluding Section V provides comments on the applications and future directions. The combination of the sol-gel process and integrated optics for micro-opto-electro-mechanical (MOEM) devices is highlighted.

\section{Amplification AND Photosensitivity in Optical WAVEGUIDES}

Silica glass is one of the most important materials in optics. With the advent of silica optical fiber for communication, the need for detailed studies of glass compositions as hosts for fiber lasers and amplifiers has gained importance. Glass hosts doped with a variety of rare-earth ions have been studied extensively in the past [3], [4]. Much of the focus has been on rare-earth materials such as neodymium, erbium, and praseodymium, because their wavelengths of operation fall in the desired windows of optical fiber communication systems. The theoretical study on Nd-doped fiber was first reported in 1985 [5]. This was soon followed by work on Er-doped fiber [6]. Studies relating to phosphate and fluoride host glasses instead of silica glass have been reported in [7], [8].

While early work was directed toward fiber lasers and amplifiers, researchers in later years attempted to explore waveguide amplifiers in integrated optics form. Fiber-based amplifiers and lasers offer advantages in terms of easy and efficient coupling to the optical transmission fiber. On the other hand, the same devices in planar integrated optic form is advantageous in terms of compactness and ability to integrate other functional elements on the same chip.

Well-known glass waveguide passive devices, such as star couplers/power splitters, could be suitably modified into amplified versions by the incorporation of a rare earth, which thereby would be useful in compensating the splitting and insertion losses [9]-[11]. Using rare-earth doped waveguides, it is also 
possible to fabricate monolithic and compact narrow linewidth single-frequency lasers by integrating coupled cavities, ring resonators, or distributed feedback mirrors. Such potentially stable laser sources would also find application at $1050 \mathrm{~nm}$ (neodymium), suitable for seeding high-power glass lasers. Finally, by employing various rare-earth ion transitions, it is possible to reach other wavelengths from the IR to the visible region of the electromagnetic spectrum by suitable up-conversion pumping schemes, as in the case of fiber lasers [12].

A good number of fabrication technologies have been suggested and explored for the fabrication of waveguide optical amplifiers [13]-[15]. Thin film deposition, diffusion, ion-exchange, flame hydrolysis and sol-gel based spin or dip coating are some of the techniques used to fabricate IO amplifiers. Another method used in the fabrication of garnet waveguides is liquid phase epitaxial growth [16]. The use of a dry silver film ion-exchange technique is reported in [17]. The propagation loss was $0.63 \mathrm{~dB} / \mathrm{cm}$ and the calculated gain was $8.8 \mathrm{~dB}$ for a $7.2-\mathrm{cm}$ long waveguide and $250-\mathrm{mW}$ pump power at $980 \mathrm{~nm}$. RF magnetron sputtering and ion beam milling were used in another study which reported a small signal gain of $3.7 \mathrm{~dB}$ with a 70-mW, 980-nm pump power [18]. Spectroscopic study of erbium doped phosphate glass planar waveguides made by either ion exchange or pulsed laser deposition has been reported [19]. A comparison of erbium-doped waveguide amplifiers is given in [20]. Er-doped lithium niobate waveguide amplifiers and other devices are reported in [21].

Amplifier efficiency is one of the important factors in practical systems. The waveguide parameters and the choice of pump wavelength have a bearing on amplifier performance. Deleterious effects include thermal and concentration quenching, and cooperative processes involving cross-relaxation and up-conversion [22], [23], [4]. For optical waveguide amplifiers, the interaction length is much shorter than that for fiber amplifiers, thereby requiring a larger active ion concentration. The larger doping level results in increased quenching. Concentration quenching is a consequence of the combined effects of ion clustering and cooperative energy transfer between ions. The microclustering in silica glasses can be improved by the use of codopants as network modifiers. In particular, it has been shown that co-doping with $\mathrm{Al}$ or $\mathrm{P}$ with $\mathrm{Nd}$ in silica glass helps to eliminate microclustering of $\mathrm{Nd}$ ions, resulting in an improved fluorescence spectrum [24]. Control of concentration quenching due to excitation migration from excited to unexcited erbium ions followed by quenching at hydroxyl impurity sites call for careful modeling and optimization. Quenching at hydroxyl sites may become a critical issue in sol-gel waveguides since they usually retain more $\mathrm{OH}^{-}$ions.

Apart from the need for efficient optical amplifiers, there are other requirements such as waveguide filters, dispersion compensators, and other grating-based devices in optical fiber communications. The demonstration of photosensitivity in germanosilicate optical fibers by Hill et al. in 1978 triggered intensive research in the direct writing of gratings in fiber and integrated optical waveguides [25]. From an experimental and analytical study, it is reported that at least two types of photorefractive effects - type I and type II-are responsible for the complicated dynamical evolution of the grating growth due to UV exposure in germonosilicate fibers [26], [27]. Besides germanosilicate fibers, photosensitivity in tin-doped phosphosilicate, tantalum-doped silica, and cerium-doped silica optical fibers have also been reported [28]-[31].

There have also been some studies of photosensitivity in planar optical waveguides: phosphorous-doped silica glass waveguides formed by flame hydrolysis [32] and Ge-doped silica waveguides fabricated by PECVD [33], [34] have been shown to exhibit photosensitivity when exposed to UV light.

Sol-gel technology is the topic of interest in this paper. Synthesis of several oxide glasses and waveguides have been known to employ the sol-gel process [35]. Of these, in terms of potential use in fiber optic communications, three compositional groups are of importance: 1) titania-silica glasses for passive devices; 2) rare-earth doped silica glasses for laser and amplifier applications; and 3) photosensitive glasses which can be used both for direct writing of channel waveguides as well as writing of Bragg gratings. Different sol-gel techniques have been employed both for bulk, as well as waveguide, applications. The sol-gel process has also been used to introduce organic molecules and enzymes in gels to fabricate various sensors.

One of the difficulties when it comes to the fabrication of thick sol-gel films required in integrated optics is that the film cracks if the deposited layer is thicker than a certain value. A sol-gel process based on the repetitive spin coating and rapid thermal annealing which overcomes this problem has been developed at Imperial College, London, U.K. The method uses a multilayer processing in which thermal annealing is done at the proper temperature after the application of each individual layer by spin coating [36], [37]. A dip-coating method along similar lines has also been reported [15]. A reflow and burial technique to form a sol-gel glass channel waveguide on silicon has also been reported [38]. Sol-gel synthesis of fluoride optical materials has been studied for IO applications [39]. Another important method involves the use of organically modified silicate system (ORMOSIL). This hybrid system uses organo-mineral precursors and the sol-gel process. This way, one can obtain UV light to directly pattern channel waveguides or other devices, bypassing the need for a mask and etching procedure [40]-[42]. Numerical simulations to understand and optimize the parameters in connection with the fabrication of channel waveguides by the sol-gel process are described in [43].

Sol-gel waveguides doped with rare-earth elements have been studied by Orignac et al. [44], [45]. A loss value of $\sim 1$ $\mathrm{dB} / \mathrm{cm}$ and a fluorescence life time of $375 \mu$ s were measured for the case of a $\mathrm{Nd}$-doped sol-gel film, while for the case of Er-doped film, the fluorescence life time was $1.78 \mathrm{~ms}$. In the case of Nd-doped sol-gel film, quenching was found to occur at concentrations above 1 at. \%. Optical properties of Er-doped sol-gel derived films have also been investigated by other groups [46]-[48]. In [48], the fluorescence lifetime of about $8 \mathrm{~ms}$, a propagation loss of $\sim 0.7 \mathrm{~dB} / \mathrm{cm}$, and a relative gain upto $1 \mathrm{~dB} / \mathrm{cm}$ were reported. Theoretical analysis of amplified spontaneous emission in dye-doped sol-gel amplifiers is recently reported [49].

Photosensitivity, Bragg grating formation, and fabrication of channel waveguides by UV exposure of sol-gel films have 
been reported [50]-[53]. Photosensitivity studies in sol-gel based waveguides have two objectives: 1) direct writing of waveguiding structures and 2) fabrication of Bragg grating in planar and channel waveguides. Both objectives can be achieved in sol-gel derived photosensitive waveguide by first photoinscribing the channel waveguide by UV exposure, and then inscribing the grating into the waveguide by a second exposure through a phase mask [51], [52]. An analysis of the grating reflectivity using a vectorial coupled-mode theory is reported in [50].

\section{ANAlysis of OptiCAl WAVEGUIDE AMPLIFYING DEVICES}

Modeling and simulation of waveguide amplifiers consists of combining waveguiding properties with laser action. One specific feature is the role of the waveguide dimensions and mode confinement. Two common methods of analysis are: 1) the rate equation approach and 2) the atomic susceptibility theory of lasers. While the former is more comprehensive, the latter provides an easy way to model the refractive index variations due to doping [3].

We present an analysis of gain characteristics using atomic susceptibility theory. With a view to study the influence of the waveguide parameters on the gain profile, its dispersion properties were determined by solving the nonlinear dispersion equation. The atomic susceptibility theory is used to obtain the gain coefficient by fitting a Gaussian curve to the absorption spectra. The modified gain characteristics were determined from the susceptibility profile and the waveguide dispersion. Alternatively, using the rate equations approach, by modeling the amplifier as a three-level system and solving for the population densities of each of the laser levels, we determined the gain coefficient. However, in the case of waveguide amplifiers, one should take into account the dependence of pumping and stimulated emission rates on the waveguide parameters. In the case of waveguide devices such as the $y$-branch or directional couplers, the Beam Propagation Method can be used to simulate the device characteristics by incorporating the amplifying effects in terms of the waveguide complex effective refractive index.

\section{A. Atomic Susceptibility Theory}

This theory [54], [55] essentially exploits the use of the density matrix description, which is a semi-classical approach, wherein the electrical field interacting with the matter is considered to be classical, while the atomic system is quantized. A full description of the complex atomic susceptibility and complex refractive index associated with the gain medium can be obtained with the model.

The starting point in this model is the analysis of the propagation of an ideal electromagnetic plane wave through an atomic medium. The terms under consideration include optical gain or loss, atomic phase shift, ohmic loss, and scattering loss.

Contribution of the lasing atoms in the atomic medium is represented by the dielectric polarization $P_{\text {as }}$. The medium properties are represented using the complex susceptibility $\chi_{\text {as }}$, which is a function of the frequency $\omega$. The approach is useful for incorporating the waveguiding properties of the gain, since the latter can be related to the imaginary part of the susceptibility. From
Maxwell's equations, we arrive at the scalar wave propagation equation containing the complex susceptibility as follows:

$$
\left[\nabla^{2}+\omega^{2} \mu \epsilon\left(1+\chi_{\text {as }}-j \sigma / \omega \epsilon\right)\right] E(x, y, z)=0
$$

where

$$
\begin{array}{ll}
E(x, y, z) & \text { phasor amplitude of any one of the vector compo- } \\
& \text { nents of } \mathbf{E} ; \\
\mu & \text { permeability; } \\
\epsilon & \text { permittivity; } \\
\sigma & \text { ohmic conductivity of the medium. }
\end{array}
$$

For propagation along the $z$ axis

$$
\left[\frac{d^{2}}{d z^{2}}+\beta^{2}\left(1+\chi_{\text {as }}-j \sigma / \omega \epsilon\right)\right] E(z)=0
$$

where the propagation constant is thus defined as $\beta=\omega(\mu \epsilon)^{1 / 2}$. The propagation factor $\Gamma$ can be written as

$$
\Gamma=j \beta\left[1+\chi^{\prime}(w)+j \chi^{\prime \prime}(w)-j \sigma / \omega \epsilon\right]^{1 / 2}
$$

where the complex atomic susceptibility is written as $\chi_{\text {as }}=$ $\chi_{\text {as }}^{\prime}+\chi_{\text {as }}^{\prime \prime}$. Thus, the complex atomic susceptibility has a resonant lineshape with frequency-dependent real and imaginary parts.

The final solution can be expressed as

$E(z, t)=\operatorname{Re}\left\{E_{o} \exp \left[-j \beta z-j \Delta \beta_{m} z-\alpha_{o} z+g_{m} z+j \omega t\right]\right\}$

The electric field varies with propagation distance and time across the homogeneous gain (or loss) medium according to (4). The first term in (4), $\beta z$, accounts for the phase variation, which can be significant over large distances (compared to $\lambda$ ). The second term in (4) is the atomic phase shift which comes into play due to the real part of the atomic susceptibility $\chi^{\prime}$. The third term is the ohmic or background loss coefficient. The term that has much significance in this work is the atomic gain coefficient, given by $g_{m}$, which results from the imaginary part of the atomic susceptibility $\chi^{\prime \prime}$, and is represented by the fourth term.

The absorption spectrum being proportional to the $\chi^{\prime \prime}(\omega)$ lineshape, it is possible to model experimental spectra to obtain $\chi^{\prime \prime}(\omega)$ at the resonant frequency $\omega_{a}$, which is taken for a strongly inhomogeneous limit to be

$$
\chi_{\text {as }}^{\prime \prime}(\omega)=\chi_{o}^{\prime \prime} \exp \left[-(4 \ln 2)\left(\frac{\omega-\omega_{a}}{\Delta \omega_{i}}\right)^{2}\right]
$$

where

$$
\chi_{o}^{\prime \prime}=\frac{1}{4 \pi^{2}} \frac{\Delta N \lambda^{3}}{\Delta \omega_{i} \tau_{\mathrm{rad}}}
$$

and $\Delta N$ is the inverted population density (atoms $/ \mathrm{cm}^{3}$ ), $\lambda$ is the absorption wavelength, $\tau_{\text {rad }}$ is the radiative decay time, and $\Delta \omega_{i}$ is the 3-dB spread in the angular frequency spectrum for the inhomogeneous case. From the above discussion, we are indeed able to observe the inherent dependence of the absorption spectra on the lineshape of $\chi^{\prime \prime}(\omega)$. This is then used to calculate the gain coefficient and, hence, the value of the absolute gain of the medium. 
The gain coefficient of the medium is given by

$$
g_{m}=\frac{1}{2} \frac{\omega \chi^{\prime \prime}(\omega)}{c}
$$

where $c$ is the velocity of light in the medium. The absolute gain is given by the following expression:

$$
G(\omega)=|E(z, t)|^{2}=\exp \left(2 g_{m} L\right)
$$

where $L$ denotes the length of the gain medium.

Assuming an absorption coefficient proportional to the atomic susceptibility, Lorentzian, Gaussian, or other line-shape functions can be used to fit the spectral variation at different peaks in experimentally measured absorption spectra. It is observed that the broadening in the case of Nd-glass sample is inhomogeneous and a Gaussian line shape function is a better approximation. From the best-fit curve, the FWHM and the gain coefficient can be calculated.

When the amplifier is in the form of a waveguide, the gain profile expression is modified by incorporating the waveguide structural parameters, such as the waveguide dimensions, the refractive index variations, and operating optical frequency. A direct way to do this is to replace the free-space (bulk) propagation constant $\omega / c$ with the waveguide mode propagation factor $\beta(\omega)$, which varies with operating frequency through the nonlinear equation representing waveguide modal dispersion. The structural parameters of the waveguide can be introduced through the $V$ parameter given by

$$
V=\frac{2 \pi}{\lambda} d \sqrt{n_{f}^{2}-n_{s}^{2}}
$$

where $d$ is the waveguide depth, and $n_{f}$ and $n_{s}$ are the refractive indices of the film and of the substrate, respectively.

The dispersion equation in the case of planar thin films for the $m$ th mode of a planar waveguide is given by

$$
\begin{aligned}
V \sqrt{1-b}-\tan ^{-1} \sqrt{b /(1-b)} & \\
& -\tan ^{-1} \sqrt{(b+a) /(1-b)}=m \pi
\end{aligned}
$$

where $a$ is the asymmetry parameter and $b$ is the normalized propagation constant, related to the propagation factor by

$$
\beta=\frac{2 \pi}{\lambda} \sqrt{n_{s}^{2}+b\left(n_{f}^{2}-n_{s}^{2}\right)}
$$

The gain coefficient for the waveguide case can now be considered as proportional to the product of two functions, $\beta(\omega)$ and $\chi^{\prime \prime}(\omega)$, which can be expressed as

$$
g_{w}(\omega)=\frac{1}{2} \beta(\omega) \chi^{\prime \prime}(\omega)
$$

The experimental fabrication and characterization of sol-gel films studied by us is presented in the next section (Section IV). The measured absorption spectrum of a typical sample showing different peaks (585 and $460 \mathrm{~nm}$ ) are shown in Fig. 1. Using this, Lorentzian and Gaussian curve fits for the peak at $585 \mathrm{~nm}$ is shown in Fig. 2. The solid curve denotes the experimental spectrum, the dashed curve denotes the Gaussian fit, and the

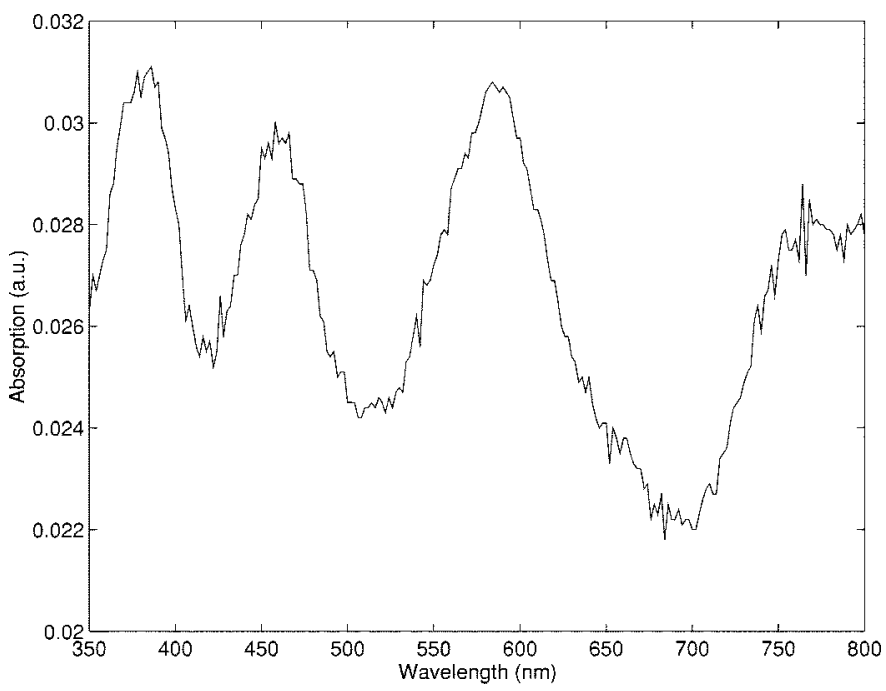

Fig. 1. Absorption spectrum of Nd-doped sol-gel waveguide sample.

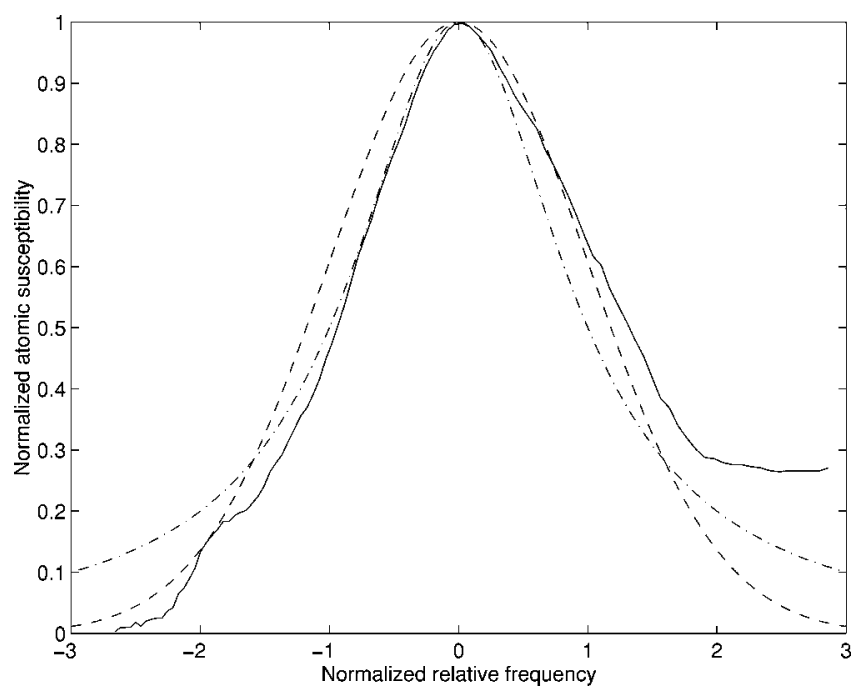

Fig. 2. Modeling of absorption spectra.

dotted-dashed curve denotes the Lorentzian fit. It is seen that the Gaussian fit is a better approximation, and can be used to calculate the gain of the $\mathrm{Nd}$-waveguide amplifier.

Next, the effect of waveguide parameters on the gain profile was studied. If we represent the change in gain profile for the bulk and waveguide cases as $\Delta g(\omega)$, then

$$
\Delta g(\omega)=g_{w}(\omega)-g_{b}(\omega)
$$

where $g_{w}(\omega)$ is the gain coefficient in the waveguide and $g_{b}(\omega)$ is the gain coefficient in bulk.

This variation for an Nd-doped sample is plotted in Fig. 3 , where change in the gain is shown against the normalized relative frequency (NRF) given by $\left(\omega-\omega_{a}\right) / \Delta \omega_{i}$ for different waveguide depths: $0.7 \mu \mathrm{m}$ (solid curve), $1.0 \mu \mathrm{m}$ (long dashed curve), and $1.2 \mu \mathrm{m}$ (short dashed curve). We find that the gain profile is modified asymmetrically. The asymmetry is more pronounced in the case of waveguides with stronger guidance. This information is useful to design waveguide amplifiers with optimal gain. 


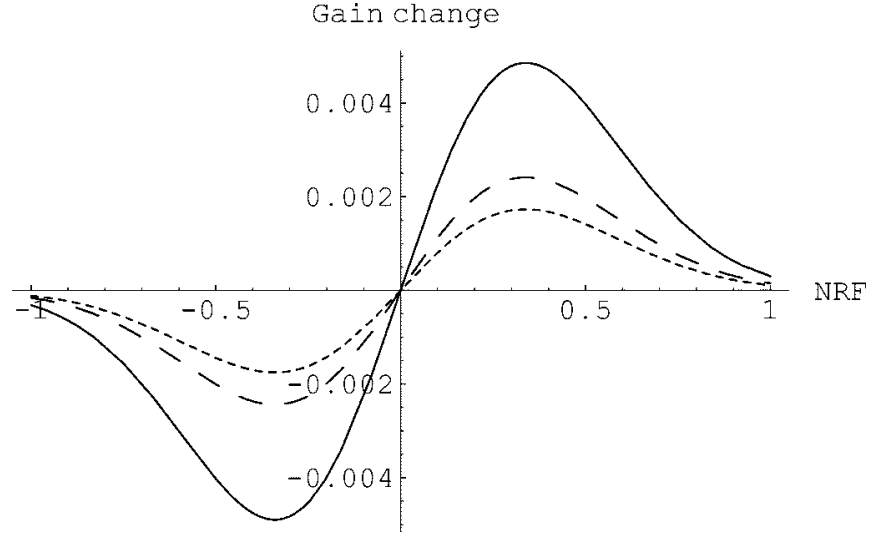

Fig. 3. Variation of change in gain $(\Delta g)$ with NRF.

\section{B. Method of Rate Equations}

The method of rate equations [3], [56], to analyze the gain in optical amplifiers, consists of two major steps: 1) calculation of the population densities of the atomic system and 2) evaluation of amplification (or attenuation) of the pump and signal beams as they propagate through the medium.

The population dynamics of a laser amplifier can be studied by considering the atomic system to be a three- or four-level system. The three-level system is a simplified and yet conceptually useful representation of an erbium laser. Atoms from the ground state are lifted to upper level 2 by pumping, from where the atoms relax to level 1 nonradiatively. Lasing takes place between upper level 1 and ground level 0 . If we denote the pumping rate, stimulated emission rate, and spontaneous decay rate as $R, W$, and $A$, respectively, then we may represent the various transitions, as:

$R_{02} \quad$ pumping rate between levels 0 and 2;

$R_{20} \quad$ stimulated emission rate between levels 2 and 0 ;

$A_{10}^{R} \quad$ spontaneous radiative decay rate;

$A_{21}^{\mathrm{NR}} \quad$ nonradiative decay rate;

$W_{01}, W_{10}$ stimulated absorption and emission rates, respectively, between levels 0 and 1 .

From the rate equations and after simplification, we get the population densities for the lasing levels under steady-state conditions as

$$
\begin{aligned}
& N_{0}=N\left[\frac{1+W_{10} \tau}{1+R \tau+W_{01} \tau+W_{10} \tau}\right] \\
& N_{1}=N\left[\frac{R \tau+W_{01} \tau}{1+R \tau+W_{01} \tau+W_{10} \tau}\right]
\end{aligned}
$$

where it is assumed that $A_{21} \gg R_{02}$ and $R_{20}, A_{10}^{R}=1 / \tau$, and $R=R_{02}$.

If a light signal of intensity, $I_{s}$ at $\lambda_{s}$ traverses a thin slice of the amplifier of thickness $d z$ and with atomic population densities, $N_{0}$ and $N_{1}$, then the intensity change is given by

$$
\frac{d I_{s}}{d z}=\left\{\sigma_{10}\left(\lambda_{s}\right) N_{1}-\sigma_{01}\left(\lambda_{s}\right) N_{0}\right\} I_{s}
$$

where $\sigma_{01}$ and $\sigma_{10}$ are the absorption and emission cross sections, respectively.

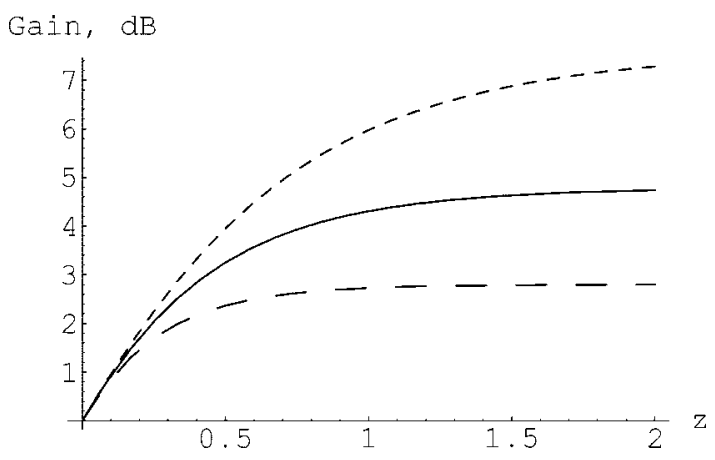

Fig. 4. Variation of gain with optical waveguide length.

The gain coefficient is obtained from the above equation and is given by

$$
g=\left\{\sigma_{10}\left(\lambda_{s}\right) N_{1}-\sigma_{01}\left(\lambda_{s}\right) N_{0}\right\}
$$

For light guided by the waveguide, the signal power coupled into the mode will have a finite spatial distribution over the waveguide plane. Defining the mode envelope as $\psi_{x, y}$, where $(x, y)$ represents the rectangular transverse coordinates, with $z$ being the direction of propagation along the waveguide, the optical power coupled into the mode $P_{s}$ results in a light intensity distribution $I(x, y)$ in the waveguide transverse plane, given by

$$
I(x, y)=P_{s} \frac{\psi_{s}(x, y)}{\int_{S} \psi_{s}(x, y) d x d y} .
$$

In terms of $P_{s}$, we can write

$$
P_{s}=\int_{s} I_{s}(x, y) d x d y .
$$

Hence, the rate at which the optical power changes as it propagates along the waveguide is

$$
\begin{aligned}
\frac{d P_{s}}{d z}=P_{s} \int_{s}\left\{\sigma_{e}\left(\lambda_{s}\right) N_{1}(x, y)-\sigma_{a}\left(\lambda_{s}\right)\right. & \left.N_{0}(x, y)\right\} \\
& \times \bar{\psi}_{s}(x, y) d x d y
\end{aligned}
$$

where $\bar{\psi}_{s}(x, y)$ is the normalized mode envelope given by

$$
\bar{\psi}_{s}(x, y)=\frac{\psi_{s}(x, y)}{\int_{s} \psi_{s}(x, y) d x d y} .
$$

The variation of gain with waveguide length for different normalized signal power coupled into the waveguide mode is shown in Fig. 4 for an erbium-doped sol-gel waveguide amplifier. The solid curve represents gain for an incident power of $P_{s 0}=1$, short dashes for $P_{s 0}=0.5$, and long dashes for $P_{s 0}=2$. The gain dependence of a waveguide amplifier in terms of length, mode field properties, input signal, and pump powers can thus be easily modeled.

\section{Integrated Optic Devices With Amplifying Dopants}

Analysis of integrate-optic devices with amplifying dopants presents several interesting features [14]. Novel devices, particularly with unity transfer functions, are possible. Here, we simulate the properties of typical integrated optic devices such as a straight waveguide, tapered waveguide, $Y$-branch, and directional coupler. The 3-D problem is first reduced to a 2-D one by 


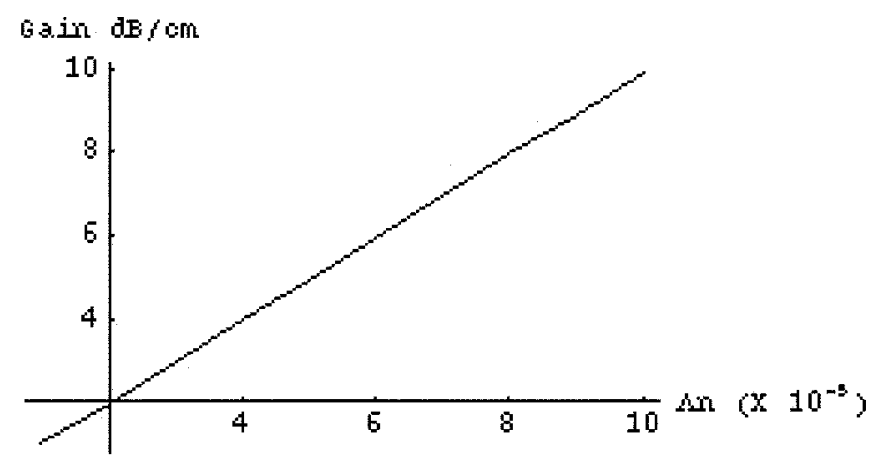

Fig. 5. Small-signal gain variation of straight waveguide.

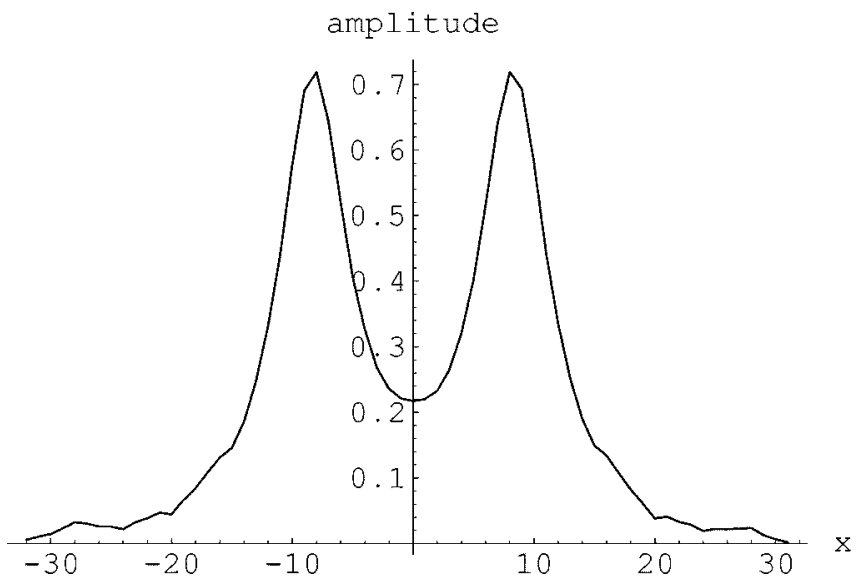

(a)

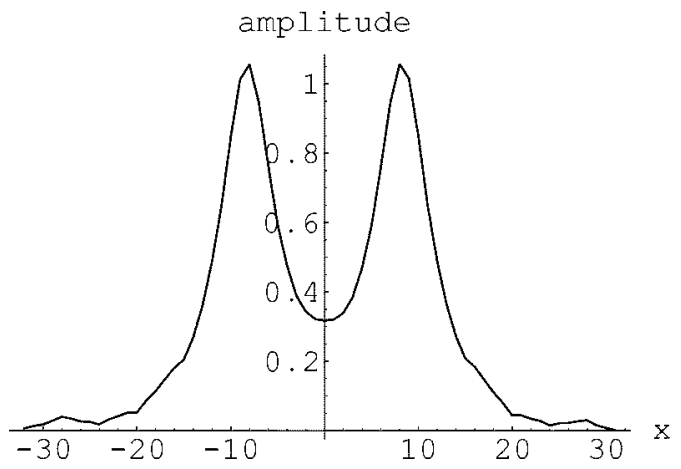

(b)

Fig. 6. Output field of a waveguide $Y$-branch: (a) without gain and (b) with gain.

using the effective index method [57] and simulations are carried out using the conventional Beam Propagation Method [58]. For this purpose, the refractive index profile of the waveguide is modified to include an imaginary part as per the susceptibility theory discussed earlier (Section III-A).

Fig. 5 shows the small-signal gain variation of a Nd-doped straight waveguide with change in effective refractive index. For a reasonably strong confinement, the gain can be as high as $10 \mathrm{~dB} / \mathrm{cm}$. Results of simulation of light propagation (in terms of normalized field amplitude) in an active waveguide $Y$-branch (lower plot) and a passive waveguide $Y$-branch without gain (upper plot) are shown in Fig. 6. Compared with a normal passive $Y$-branch, we see that the output power is amplified by $3 \mathrm{~dB}$ in each of the output ports. The refractive index of the $Y$-branch

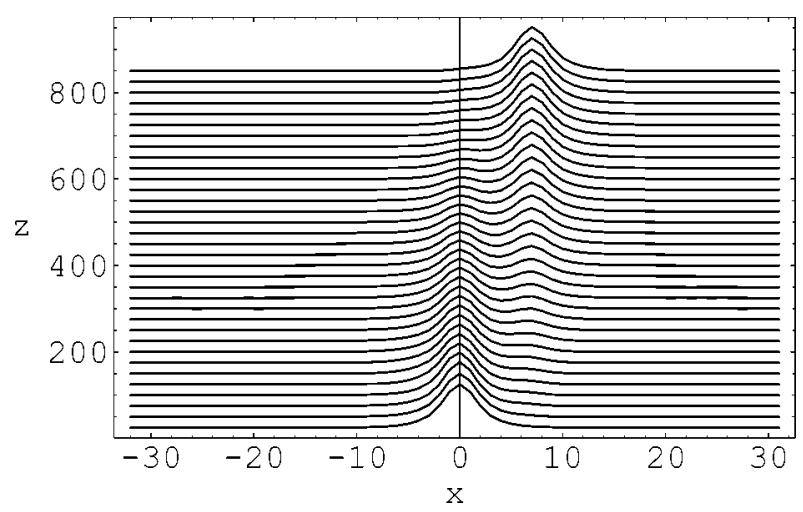

(a)

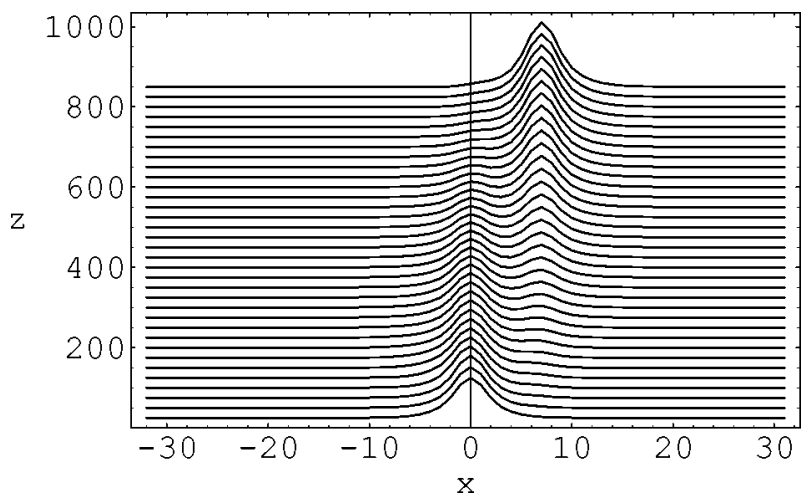

(b)

Fig. 7. Simulation of propagation in a directional coupler: (a) without gain and (b) with gain.

is optimized to obtain a unity gain. Simulations in the case of a directional coupler designed for the cross-over state (Fig. 7) show that in addition to amplification, the cross-over coupling length is altered. The parameters (typically) used for the simulations are: operating wavelength $\lambda=1.3 \mu \mathrm{m}$, substrate refractive index $n_{s}=1.455$, waveguide refractive index $n_{f}=1.4846$, waveguide height $h=2 \mu \mathrm{m}$, waveguide width $=3.4 \mu \mathrm{m}$, and imaginary part of the effective refractive index $\Delta n_{I} \sim 10^{-5}$. These studies help to optimize the gain characteristics of integrated optic devices of unity gain or any desired gain value for a given set of waveguide parameters.

\section{EXPERIMENTAL WORK}

\section{A. Fabrication of Sol-Gel Waveguides}

Fabrication of composite planar waveguides on glass substrates doped with $\mathrm{Nd}^{3+}$ and $\mathrm{Er}^{3+}$ ions is easily achieved using the sol-gel method [59]. We have used a process which consists of forming a multilayer film by spin-coating several thin layers and thermal annealing after the application of each individual layer [36], [37]. Planar waveguides on glass (refractive index $=1.517$ ) were prepared using the sol-gel process. Tetraethyl orthosilicate (TEOS) and titanium (IV) isopropoxide (TPOT) were used as precursors in the molar ratio of 80:20. The recipe for sol was as follows: TEOS $(5.89 \mathrm{ml})$ was first mixed with absolute ethanol $(25 \mathrm{ml})$ and partially hydrolyzed by the addition of $0.1 \mathrm{~N} \mathrm{HCl}$ keeping the water:alkaloid ratio $(R)$ at one for $2 \mathrm{~h}$ at $300 \mathrm{~K}$ in a magnetic stirrer. TPOT $(2 \mathrm{ml})$ was separately hydrolyzed in absolute ethanol $(25 \mathrm{ml})$ containing a few 
drops of concentrated $\mathrm{HNO}_{3}$ and stirred for $30 \mathrm{~min} . \mathrm{HNO}_{3}$ was added to keep the ethanolic solution of TPOT clear, avoiding the formation of a cloudy precipitate of titanium hydroxide. Both the alcohol solutions of TEOS and TPOT were then mixed and stirred for about $1 \mathrm{~h}$.

In the case of $\mathrm{Nd}^{3+}$, neodymium trioxide $\left(\mathrm{Nd}_{2} \mathrm{O}_{3}\right)$ was converted into nitrate form using concentrated $\mathrm{HNO}_{3}$; this was then used as the dopant with a concentration of 2.5 at. \%, during the hydrolysis step of the alkaloid mixture. An ethanolic solution of phosphorous pentaoxide (Nd:P::1:10) was also added to the sol to avoid the clustering of $\mathrm{Nd}$ atoms during the glass formation.

The sol was aged for $24 \mathrm{~h}$ and was spin-coated on clean glass substrates. The coating was done for $30 \mathrm{~s}$ at $3000 \mathrm{rpm}$ on a Headway Research Inc., spinner. Soon after coating, the samples were annealed at a temperature of $500{ }^{\circ} \mathrm{C}$ for $15 \mathrm{~min}$. Typically, five layers on each sample were formed using this procedure.

A similar process was adopted for the doping with cerium [60]. The sol-gel preparation involves the hydrolysis and condensation of tetraethyl orthosilicate with water and dilute hydrochloric acid in an alcoholic medium. To this, an ethanolic solution of phosphorus pentoxide is added and finally cerium is added in the form of cerium nitrate. The mixture was agitated for $2 \mathrm{~h}$ for complete dispersion of cerium nitrate into the gel. $\mathrm{P}_{2} \mathrm{O}_{5}$ was used as a codopant to enhance the photosensitivity.

As another example, preparation of an organic-inorganic composite material using the sol-gel process is given below [61]. The composite sol-gel was prepared using polyvinylpyrolidone (PVP) as the organic constituent, while TEOS and TPOT in the molar ratio of 80:20 formed the inorganic constituent. The ratio of the organic to inorganic constituent was 50:50 (wt.\%).

\section{B. Characterization of Sol-Gel Films and Waveguides}

The sample waveguides as described above were excited by the prism-coupling method [57]. It was found that they supported 1-3 modes, as evidenced by $m$-line measurements at $\lambda=633 \mathrm{~nm}$. The refractive index difference between the waveguide and the glass substrate was typically 0.03 (for Nd-doped samples) and the thickness of the coating per layer was about $200 \mathrm{~nm}$. Using a surface-scattering measurement technique, the propagation loss was found to be $2 \mathrm{~dB} / \mathrm{cm}$. Spectral characterization and gain measurements of the samples were done to study their amplification properties.

Broadening of atomic transition line shape was studied using absorption spectra. We have observed that the results obtained for spin-coated sol-gel based Nd-glass optical waveguides are in good agreement with the studies conducted in bulk materials.

Fig. 8 shows the experimental setup used to characterize the waveguide amplifiers. The gain measurement was performed using a dye laser (Quanta Ray DCR-3) as the pump source at a wavelength of $575 \mathrm{~nm}$. A small signal at $1060 \mathrm{~nm}$ from a $\mathrm{Nd}$ :YAG laser was used to measure the signal gain. A filter was used at the output to cut-off wavelengths less than $800 \mathrm{~nm}$. The sample length was typically $1 \mathrm{~cm}$ and the launched signal power was $1-2 \mu \mathrm{W}$. The measured gain, on average, was $14 \mathrm{~dB} / \mathrm{cm}$. The calculated gain using the atomic susceptibility theory was $17 \mathrm{~dB} / \mathrm{cm}$. Taking into account the propagation loss, it is seen

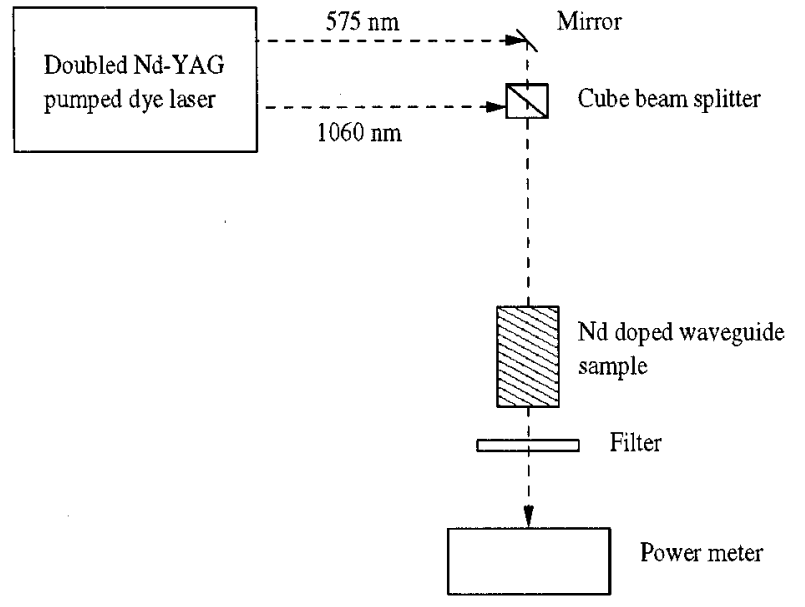

Fig. 8. Experimental setup to characterize the waveguide amplifiers.

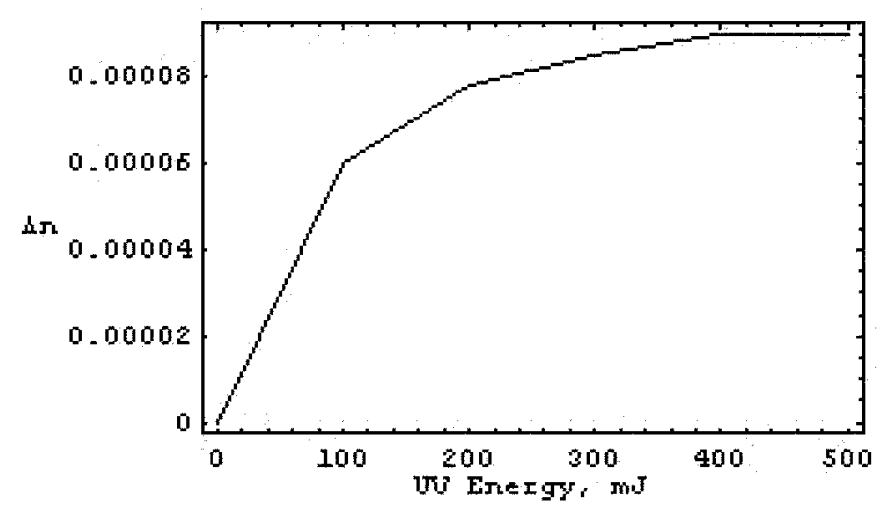

Fig. 9. Refractive index change with different UV energy in a Ce-doped solgel waveguide.

that there is a reasonable agreement between the predicted and measured values [62]. The gain in the case of Nd-doped waveguides is high compared to the gain in EDWA, which is about $4.5 \mathrm{~dB} / \mathrm{cm}$ [20]. Quenching due to $\mathrm{OH}^{-}$is one of the reasons for the use of lower doping concentration and hence a lower gain. It is known that Er-doped gain media are more sensitive to $\mathrm{OH}$ ion quenching than Nd-doped material.

\section{Photosensitive Waveguides: Bragg Gratings}

Gratings formed on waveguides have many applications in integrated optics. Sol-gel waveguide films doped with elements such as cerium and germanium exhibit photosensitivity when exposed to UV radiation. This can be used to write Bragg gratings in optical waveguides. The permanent change in the refractive index on exposure to light can be explained using either the color center model or densification model. Theoretical analysis of the characteristics of waveguide gratings can be done using the coupled-mode theory [57], [63].

Photo-induced refractive index changes are well known in germano-silicate fibers [25]. In germania-doped devices, the photosensitivity depends on the population of $\mathrm{GeO}$ defects, which is difficult to control during fabrication. On the other hand, $\mathrm{Ce}$ can be easily photo-ionized to $\mathrm{Ce}^{3+}$, which leads to the formation of color centers in both crystalline and 


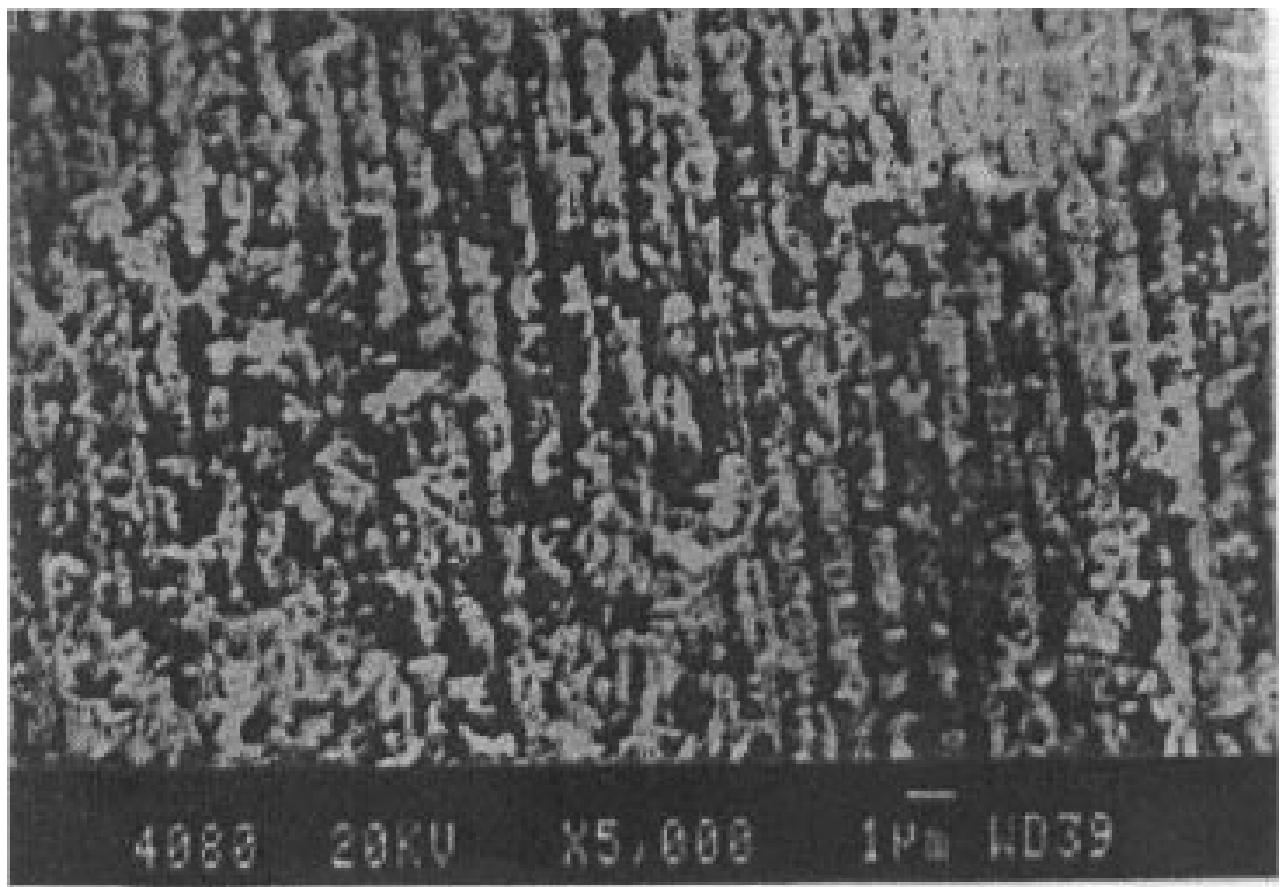

Fig. 10. Scanning electron microscope photograph of a Ce-doped sol-gel waveguide grating.

amorphous host materials. In the case of rare-earth doped sol-gel waveguides, $\mathrm{Ce}^{3+}$ doping also reduces the formation of clusters resulting from the incorporation of high concentration of rare-earth ions. The absorption spectra of cerium-doped samples show a peak near $296 \mathrm{~nm}$, which is due to $\mathrm{Ce}^{3+}$ color centers and corresponds to a 4f-5d electronic dipole transition. The position of the peak is highly dependent on the glass composition. Due to this electronic transition, exposure to light leads to permanent change in the refractive index.

Cerium ions exist in two ionic states, cerous $\left(\mathrm{Ce}^{3+}\right)$ and ceric $\left(\mathrm{Ce}^{4+}\right)$ states. The equilibrium in the two states depends on basicity of glass matrix and glass preparation conditions. A photoinduced absorption change in cerium doped sol-gel waveguide has been attributed to $\mathrm{Ce}^{3+}$ ions which are photoionized to form $\left(\mathrm{Ce}^{4+}\right)$ ions on UV exposure [30], [31].

The sample waveguides as fabricated in Section IV-A was exposed to UV light $(248 \mathrm{~nm}$ ) from a $\mathrm{KrF}$ excimer laser that delivered $0.2 \mathrm{~J}$ of energy per 20 -ns pulse in a $30 \mathrm{~mm} \times 10 \mathrm{~mm}$ rectangular beam. The refractive index change for different UV exposure energies is plotted in Fig. 9, which clearly shows that the index change is nonlinear and saturation occurs around $0.4 \mathrm{~J}$. Next, the phase mask technique was employed to imprint gratings on the waveguide samples. Exposure of the waveguide to the pulsed excimer laser $(248 \mathrm{~nm})$ through the phase mask resulted in a refractive index modulated grating. A scanning electron microscope photograph of a UV-exposed Ce-doped film is shown in Fig. 10. Grating formation due to densification in the exposed region can be observed in the figure. A typical refractive index change of $2.0 \times 10^{-5}$ was obtained. The waveguide with grating was tested using $\mathrm{He}-\mathrm{Ne}$ laser for diffraction of light. The diffraction efficiency was typically about $40 \%$. The stability of the grating was tested by a heating arrangement. A decrease in diffraction efficiency by $0.1 \mathrm{~dB} /{ }^{\circ} \mathrm{C}$ was observed in the temperature range of $40^{\circ} \mathrm{C}-120^{\circ} \mathrm{C}$.

\section{CONCLUSION}

The sol-gel process is an effective method of fabrication of integrated optical waveguides and devices, particularly due to the ease of incorporating various dopants. In this paper, the fabrication of sol-gel based integrated optic waveguides with neodymium, erbium, and cerium was presented, along with their application to specific devices, namely optical amplifiers and Bragg gratings. Results of analysis, simulation, and experimental work are given. In recent years, there has been rapid progress in the fabrication and characterization of sol-gel based IO waveguides and devices, with and without active dopants. Several research groups across the world have proven core competence in this area. However, to our knowledge there has been no report of packaged devices ready for commercial exploitation. Long-term stability and improved performance along with packaging issues need to be addressed soon so that the inherent potential in terms of economy and flexibility offered by the sol-gel technology becomes practical. With increasing interest in micro-electro-mechanical systems for sensors, smart structures, and communications applications, the sol-gel process attains further importance in developing optical MEMS where optical devices could be controlled by mechanical structures [64]. The sol-gel process thus presents a simple and effective route to conceive and develop novel integrated optic devices.

\section{ACKNOWLEDGMENT}

The authors would like to acknowledge the contributions of Mr. S. R. Natarajan, Dr. G. M. Hegde, and Mr. A. A. Kumar. Suggestions and corrections provided by both the reviewers and the Associate Editor have helped to improve the presentation of this paper, and are gratefully acknowledged. Mr. T. Venugopal and Mr. P. K. Pattnaik have assisted in the preparation of this manuscript. 


\section{REFERENCES}

[1] Opt. Eng., vol. 37, no. 4, Apr. 1998.

[2] S. R. Natarajan, "Theoretical and Experimental Studies on Optical Amplification in Sol-Gel Films," Ph.D. dissertation, Indian Institute of Science, Bangalore, India, Apr. 2000.

[3] E. Desurvire, Erbium Doped Fiber Amplifiers. New York: Wiley Interscience, 1994.

[4] W. J. Miniscalco, "Erbium doped glasses for fiber amplifiers at 1500 nm," J. Lightwave Technol., vol. 9, pp. 234-250, Feb. 1991.

[5] M. J. F. Digonnet and C. J. Gaeta, "Theoretical analysis of optical fiber laser amplifiers and oscillators," Appl. Opt., vol. 24, no. 3, pp. 333-342, 1985.

[6] R. Z. Mears, L. Reekie, I. M. Jallncey, and D. Payne, "Low-noise erbium-doped fiber amplifier operating at $1.54 \mu \mathrm{m}$," Electron. Lett., vol. 23, no. 19, pp. 1026-1028, 1987.

[7] S. Jiang, M. Myers, and N. Peyghambarian, "Er ${ }^{3+}$-doped phosphate glasses and lasers," J. Non-Crystalline Solids, vol. 239, pp. 143-148, 1998.

[8] Z. Meng, T. Yoshimura, Y. Nakata, N. J. Vasa, and T. Okada, "Improvement of fluorescence characteristics of $\mathrm{Er}^{3+}$-doped fluoride glass by $\mathrm{Ce}^{3+}$ codoping," Jpn. J. Appl. Phys., pt. 2, vol. 38, no. 12A, pp. L1409-L1411, 1999.

[9] P. Camy, J. E. Roman, F. W. Willems, M. Hempstead, J. C. Vander Plaats, C. Prel, A. Beguin, A. M. J. Koonen, J. S. Wilkinson, and C. Lerminiaux, "Ion-exchanged planar lossless splitter at $1.5 \mu \mathrm{m}$," Electron. Lett., vol. 32, no. 4, pp. 321-322, 1996.

[10] D. Barbier, M. Rattay, F. S. Andre, G. Clauss, M. Trouillon, A Kevorkian, J. M. P. Delavaux, and E. Murphy, "Amplifying four-wavelength combiner, based on erbium/ytterbium-doped waveguide amplifiers and integrated splitters," IEEE Photon. Technol. Lett., vol. 9, pp. 315-317, Mar. 1997.

[11] R. R. A. Syms, "Advances in channel waveguide lithium niobate integrated optics," Opt. Quantum Electron. Lett., vol. 20, pp. 189-213, 1988.

[12] J. Y. Allain, M. Monerie, and H. Poignant, "Room temperature cw tunable green upconversion holmium fiber laser," Electron. Lett., vol. 26, pp. 261-263, 1990.

[13] S. I. Najafi, Ed., Introduction to Glass Integrated Optics. Norwood, MA: Artech House, 1993.

[14] O. Lumholt, A. B. Jarklev, T. Rasmussen, and C. Lester, "Rare earth-doped integrated glass components: Modeling and optimization," J. Lightwave Technol., vol. 13, pp. 275-262, 1995.

[15] L. Yang, S. S. Saavedra, N. R. Armstrong, and J. Hayes, "Fabrication and characterization of low-loss, sol-gel planar waveguides," Anal. Chem. vol. 66, no. 8, pp. 1254-1260, 1994.

[16] M. Yamaga, K. Yusa, and Y. Miyazaki, "Theoretical analysis of waveguide laser amplifier using Nd doped garnet crystalline thin film," IEICE Trans. Electron., vol. E-69, no. 9, pp. 956-967, 1996.

[17] T. Ohtsuki and N. Peyghambarian, "Gain characteristics of a high concentration $\mathrm{Er}^{3+}$-doped phosphate glass waveguide," J. Appl. Phys., vol. 78 , no. 6, pp. 3617-3621, 1995

[18] R. N. Ghosh, J. Shmulovich, C. F. Kane, M. R. X. de Barros, G. Nykolak, A. J. Bruce, and P. C. Becker, "8-mW threshold $\mathrm{Er}^{3+}$-doped planar waveguide amplifier," IEEE Photon.Technol. Lett., vol. 8, pp. 518-520, Apr. 1996.

[19] G. C. Righini, S. Capecchi, S. Pelli, and A. Verciani, "Erbium doped glass waveguides for integrated optical amplifiers and lasers," in Proc. Int. Conf. Fiber Optics and Photonics (PHOTONICS'96), Madras, India, 1996, pp. 917-922.

[20] E. Barbier and R. L. Hyde, "Erbium doped glass waveguide devices," in Integrated Optical Circuits and Components: Design and Applications, E. J. Murphy, Ed. New York: Marcel-Dekker, 1999, ch. 5

[21] W. Sohler and H. Suche, "Erbium doped lithium niobate waveguide devices," in Integrated Optical Circuits and Components: Design and Applications, E. J. Murphy, Ed. New York: Marcel-Dekker, 1999, ch. 6 .

[22] E. Delevaque, T. Georges, M. Monerie, P. Lamouler, and J. F. Bayon, "Modeling of pair-induced quenching in erbium-doped silicate fibers," IEEE Photon. Technol. Lett., vol. 5, pp. 73-75, Jan. 1993.

[23] E. Snoeks, G. N. van den Hven, and A. Polman, "Optimization of an Er-doped silica glass optical waveguide amplifier," IEEE J. Quantum Electon., vol. 32, pp. 1680-1684, Sept. 1996.

[24] K. Arai, H. Namikawa, K. Kumata, and T. Honda, "Aluminum or phosphorus co-doping effects on the fluorescence and structural properties of neodymium-doped silica glass," J. Appl. Phys., vol. 59, no. 10, pp. $3430-3436,1986$.
[25] K. O. Hill, Y. Fujii, D. C. Johnson, and B. S. Kawasaki, "Photosensitivity in optical fiber waveguides: Application to reflection filter fabrication," Appl. Phy. Lett., vol. 32, no. 10, pp. 647-649, 1978.

[26] W. X. Xie, P. Niay, P. Bernage, M. Douay, J. F. Bayon, T. Georges, M. Monerie, and B. Poumellec, "Experimental evidence of two types of photorefractive effects occurring during photoinscriptions of Bragg gratings within germanosilicate fibers," Opt. Сoтmun., vol. 104, pp. 185-195, 1993.

[27] M. Douay, W. X. Xie, T. Taunay, P. Bernage, P. Niay, P. Cordier, B. Poumellec, L. Dong, J. F. Bayon, H. Poignant, and E. Delevaque, "Densification involved in the UV based photosensitivity of silica glasses and optical fibers," J. Lightwave Technol., vol. 15, pp. 1329-1342, Aug. 1997.

[28] L. Dong, J. L. Cruz, J. A. Tucknott, L. Reekie, and D. N. Payne, "Strong photosensitive gratings in tin-doped phosphosilicate optical fibers," Opt. Lett., vol. 20, no. 19, pp. 1982-1984, 1995.

[29] L. Dong, J. L. Archambault, E. Taylor, M. P. Roe, L. Reekie, and P. St. J. Russell, "Photosensitivity in tantalum-doped silica optical fibers," $J$. Opt. Soc. Amer. B, vol. 12, no. 9, pp. 1747-1750, 1995.

[30] L. Dong, P. J. Wells, D. P. Hand, and D. N. Pyne, "Photosensitivity in $\mathrm{Ce}^{3+}$-doped optical fibers," J. Opt. Soc. Amer. B, vol. 10, pp. 89-93, 1993.

[31] L. Dong, J. L. Archambault, L. Reekie, P. St. J. Russel, and D. N. Pyne, "Bragg gratings in $\mathrm{Ce}^{3+}$ doped fibers written by a single excimer pulse," Opt. Lett., vol. 18, pp. 661-663, 1993.

[32] B. Malo, J. Albert, F. Bilodeau, T. Kitagawa, D. C. Johnson, K. O. Hill, K. Hittori, Y. Hibino, and S. Gujrathi, "Photosensitivity in phosphorusdoped silica glass and optical waveguides," Appl. Phys. Lett., vol. 65 , no. 4, pp. 394-396, 1994.

[33] M. Svalgaard, C. V. Poulsen, A. Bjarklev, and O. Poulsen, "Direct UV writing of buried singlemode channel waveguides in Ge-doped silica films," Electron. Lett., vol. 30, no. 17, pp. 1401-1403, 1994.

[34] J. Canning, M. G. Sceats, and S. Flaming, "Grating structures with phase mask period in silica-on-silicon planar waveguides," Opt. Commun., vol. 171, pp. 213-217, 1999.

[35] D. Ganguli, "Sol-gel glasses: Some recent trends," Bull. Mater. Sci., vol. 18 , pp. 47-52, 1995.

[36] A. S. Holmes, R. R. A. Syms, M. Li, and M. Green, "Fabrication of buried channel waveguides on silicon substrates using spin-on glass," Appl. Opt., vol. 32, no. 25, pp. 4916-4921, 1993.

[37] R. R. A. Syms, V. Schneider, W. Huang, and A. S. Holmes, "Low loss achieved in sol-gel based silica-on-silicon integrated optics using borophosphosilicate glass," Electron. Lett., vol. 31, no. 21, pp. 1833-1834, 1995.

[38] R. R. A. Syms and A. S. Holmes, "Reflow and burial of channel waveguides formed in sol-gel glass on Si substrates," IEEE Photon. Technol. Lett., vol. 5, pp. 1077-1079, Sept. 1993.

[39] J. Ballato, R. E. Riman, and E. Snitzer, "Sol-gel synthesis of fluoride optical materials for planar integrated photonic applications," J. NonCryst. Solids, vol. 213 \& 214, pp. 126-136, 1997.

[40] X. M. Du, T. Touam, L. Degachi, J. L. Guilbault, M. P. Andrews, and S. I. Najafi, "Sol-gel waveguide fabrication parameters: An experimental investigation," Opt. Eng., vol. 37, no. 4, pp. 1101-1104, 1998.

[41] A. Fardad, M. Andrews, G. Milova, A. M. Tabrizi, and I. Najafi, "Fabrication of ridge waveguides: A new sol-gel route," Appl. Opt., vol. 37, no. 2, pp. 2429-2434, 1998.

[42] M. A. Fardad and M. Fallahi, "Organic-inorganic materials for integrated optoelectronics," Electron. Lett., vol. 34, no. 20, pp. 1940-1941, 1998.

[43] J. Porque, P. Coudray, Y. Moreau, and P. Etienne, "Propagation in sol-gel channel waveguides: Numerical and experimental approaches," Opt. Eng., vol. 37, no. 4, pp. 1105-1110, 1998.

[44] X. Orignac, D. Barbier, X. M. Du, and R. M. Ahmedia, "Fabrication and characterization of sol-gel planar waveguides doped with rare-earth ions," Appl. Phys. Lett., vol. 69, no. 12, pp. 895-897, 1996.

[45] X. Orignac and R. M. Almedia, "Silica-based sol-gel optical waveguides on silicon," in Proc. IEE Optoelectronics, vol. 143, 1996, pp 287-292.

[46] A. Bahtat, M. Bouazaoui, M. Bahtat, and J. Mugnier, "Fluorescence of $\mathrm{Er}^{+3}$ ions in $\mathrm{TiO}_{2}$ planar waveguides prepared by a sol-gel process," Opt. Commun., vol. 111, pp. 55-60, 1994.

[47] C. Strohhofer, J. Fick, H. C. Vasconceleos, and R. M. Almeida, "Active optical properties of Er-containing crystallites in sol-gel derived glass films," J. Non-Cryst. Solids, vol. 226, pp. 182-191, 1998.

[48] E. M. Yeatman, M. M. Ahmad, O. McCarthy, A. Vannucci, P. Gastaldo, D. Barbier, D. Mongardien, and C. Moronvalle, "Optical gain in Er-doped $\mathrm{SiO}_{2}-\mathrm{TiO}_{2}$ waveguides fabricated by the sol-gel technique," Opt. Commun., vol. 164, pp. 19-25, 1999. 
[49] M. Roth, A. Hardy, and S. Ruschin, "Amplified spontaneous emmision in dye-doped sol-gel amplifiers," IEEE J. Quantum Electron., vol. 37, pp. 189-198, Feb. 2001.

[50] T. Touam, M. Du, M. A. Fardad, M. P. Andrews, and S. I. Najafi, "Sol-gel waveguides with Bragg grating," Opt. Eng., vol. 37, no. 4, pp. 1136-1142, 1998.

[51] S. I. Najafi, T. Touam, R. Sara, M. P. Andrews, and M. A. Fardad, "Sol-gel glass waveguide and grating on silicon," J. Lightwave Technol., vol. 16, pp. 1640-1646, Sept. 1998.

[52] M. A. Fardad, T. Touam, P. Meshkinfam, R. Sara, X. M. Du, M. P. Andrews, and S. I. Najafi, "UV-light imprinted Bragg grating in sol-gel ridge glass waveguide with almost $100 \%$ reflectivity," Electron. Lett., vol. 33, no. 12, pp. 1069-1070, 1997.

[53] Y. Moreau, P. Arguel, P. Courdary, P. Etienne, J. Porque, and P. Signoret, "Direct printing of gratings on sol-gel layers," Opt. Eng., vol. 37, no. 4, pp. $1130-1135,1998$

[54] E. Desurvire, "Study of the complex atomic susceptibility of erbiumdoped fiber amplifiers," J. Lightwave. Technol., vol. 8, pp. 1517-1527, Oct. 1990.

[55] A. E. Siegman, Lasers: Oxford University Press, 1986

[56] A. Yariv, Optical Electronics. New York: CBS College Publishing, 1985.

[57] H. Nishihara, M. Haruna, and T. Suhara, Optical Integrated Circuits. New York: McGraw-Hill, 1989.

[58] C. R. Pollock, Fundamentals of Optoelectronics. Chicago, IL: Irwin, 1995.

[59] S. R. Natrajan, A. V. Rammurthi, A. Selvarajan, M. Muthuraman, and K. C. Patil, "Amplification of light in sol-gel based Nd-glass waveguides," in Proc. SPIE Conf. Optoelectronics and High Power Lasers and Applications, Photonics West, San Jose, CA, 1998.

[60] A. A. Kumar, G. M. Hegde, T. K. Kumar, and A. Selvarajan, "Bragg grating on cerium doped sol-gel waveguides for sensor applications," in Proc. Int. Conf. Smart Materials, Structures and Systems, Bangalore, India, 1999, pp. 239-243.

[61] S. R. Natarajan, M. J. Joseph, T. Srinivas, and A. Selvarajan, "Fabrication of rare earth doped sol-gel based composite planar waveguides on glass," in Proc. Int. Conf. Applications of Photonics Technology, vol. 3, Ottawa, ON, Canada, 1998, pp. 371-373.

[62] A. Selvarajan and S. R. Natarajan, "Optical amplification in sol-gel based waveguides," in Proc. Int. Conf. Optics and Lasers, vol. 1, Dehradun, India, 1998, pp. 438-444.

[63] T. Tahar, X. M. Du, F. Amir, M. P. Andrews, and I. S. Najafi, "Sol-gel waveguides with Bragg grating," Opt. Eng., vol. 37, no. 4, pp. 1136-1142, 1998.
[64] A. Selvarajan, P. K. Pattnaik, V. M. Gupta, and T. Srinivas, "Micro-opto-electro-mechanical (MOEM) vibration sensor," in Proc. SPIE Smart Structures and Materials: Smart Electronics and $M E M S$, vol. 3990, Newport Beach, CA, Mar. 2000, pp. 78-85.

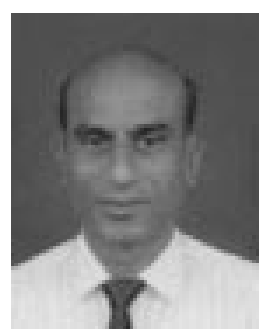

A. Selvarajan (M'01) received the M.Sc. degree in physics from Annamalai University, India, in 1964, and the Ph.D. degree from the Indian Institute of Science, Bangalore, in 1970.

$\mathrm{He}$ is a Professor in the Department of Electrical Communication Engineering, Indian Institute of Science. He has been a Visiting Research Scientist at OSC of the University of Arizona, the University of Upsala of Sweden, the Technical University of Denmark, University College of London, and the International Center for Theoretical Physics, Trestie, Italy. He has authored over 160 publications in the areas of optics and spectroscopy, holography, acousto-optics, fiber optics, and integrated opitcs. His current research interests are in the areas of integrated optic components for optical communication systems and micro-opto-electro-mechanical systems for sensors and smart structure applications.

Dr. Selvarajan is a member of the International Society for Optical Engineering (SPIE), the Optical Society of India, the Institute of Electronics and Telecommunication Engineers (IETE) of India, and the Institute of Smart Structures and Systems (ISSS).

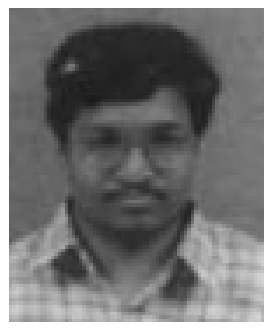

T. Srinivas (S'93-M'96) received the M.E. (integrated) and $\mathrm{Ph} . \mathrm{D}$. degrees from the Indian Institute of Science, Bangalore, in 1987 and 1993, respectively.

$\mathrm{He}$ is an Assistant Professor in the Department of Electrical Communication Engineering, Indian Institute of Science. During 1992-1996, he was a postdoctoral Research Fellow at Toyohashi University of Technology, Japan. His research interests include integrated optics and technology.

Dr. Srinivas is a member of the IEEE Lasers and Electro-Optics Society (LEOS), the Society of Industrial and Applied Mathematics (SIAM), the Institute of Electronics, Information and Communication Engineers (IEICE) of Japan, and the Optical Society of India. 